\title{
Shape phase transition and shape coexistence in the Bose- Fermi system
}

\author{
Yu Zhang ${ }^{1, \star, \star \star}$, Wenting Dong ${ }^{1}$, and $\mathrm{He}$ Jiang $^{1}$ \\ ${ }^{1}$ Department of Physics, Liaoning Normal University, Dalian, 116029, China
}

\begin{abstract}
A classical analysis of shape phase transition and shape coexistence in oddeven nuclei has been carried within the interacting boson-fermion model. The results indicate that shape coexistence can be taken as a signature of shape phase transition in odd-even nuclei.
\end{abstract}

\section{Introduction}

Shape phase transitions (SPTs) in odd-even nuclei have received some attentions in recent years [110]. Evidences of the spherical to prolate SPT and the associated shape/phase coexistence have been clearly identified in the odd Sm nuclei [8]. A theoretical tool suitable for analyzing the SPTs in odd-even nuclei is the interacting boson-fermion model (IBFM) [11], in which odd-even nucleus is approximately considered as the Bose-Fermi system with an even-even core (boson) coupled to a single particle (fermion). Previous investigations reveal that the effects of single particle may influence different type of SPT in different way [4-6]. In this work, we will present a classical analysis of the effects of single particle on the spherical to prolate SPT within the framework of IBFM. Particularly, we will emphasize how the presence of an odd particle can influence the shape/phase coexistence in this type of SPT.

\section{Classical analysis of the spherical to prolate SPTs}

In the following discussion, an IBFM Hamiltonian describing the system with the even-even core coupled to a single fermion via quadrupole-quadrupole interaction is adopted and given as

$$
\hat{H}=\varepsilon\left[(1-\eta) \hat{n}_{d}-\frac{\eta}{4 N} \hat{Q}_{B F} \cdot \hat{Q}_{B F}\right],
$$

where $\hat{n}_{d} \equiv \sum_{u} d_{u}^{\dagger} d_{u}$ and $\hat{Q}_{B F} \equiv \hat{Q}_{B}^{\chi}+\hat{q}_{F}$ with $\hat{Q}_{B}^{\chi}=\left(d^{\dagger} s+s^{\dagger} \tilde{d}\right)^{(2)}+\chi\left(d^{\dagger} \tilde{d}\right)^{(2)}$ and $\hat{q}_{F}=\left(a_{j}^{\dagger} \tilde{a}_{j}\right)^{(2)}$. Besides, $\eta$ and $\chi$ are the control parameters with $\eta \in[0,1]$ and $\chi \in[-\sqrt{7} / 2,0]$, and $\varepsilon$ is a scale factor which is set as 1 for convenience. One can prove that the spherical to prolate SPTs may occur at $\eta_{c}=\frac{28 N}{56(N-1)+\chi^{2}(5+2 N)}$ with $\chi \in[-\sqrt{7} / 2,0)$ if only the even-even core corresponding to $\hat{Q}_{B F}=\hat{Q}_{B}^{\chi}$ in

${ }^{\star}$ Support from National Natural Science Foundation of China (11375005)

$\star \star$ e-mail: dlzhangyu_physics@163.com 

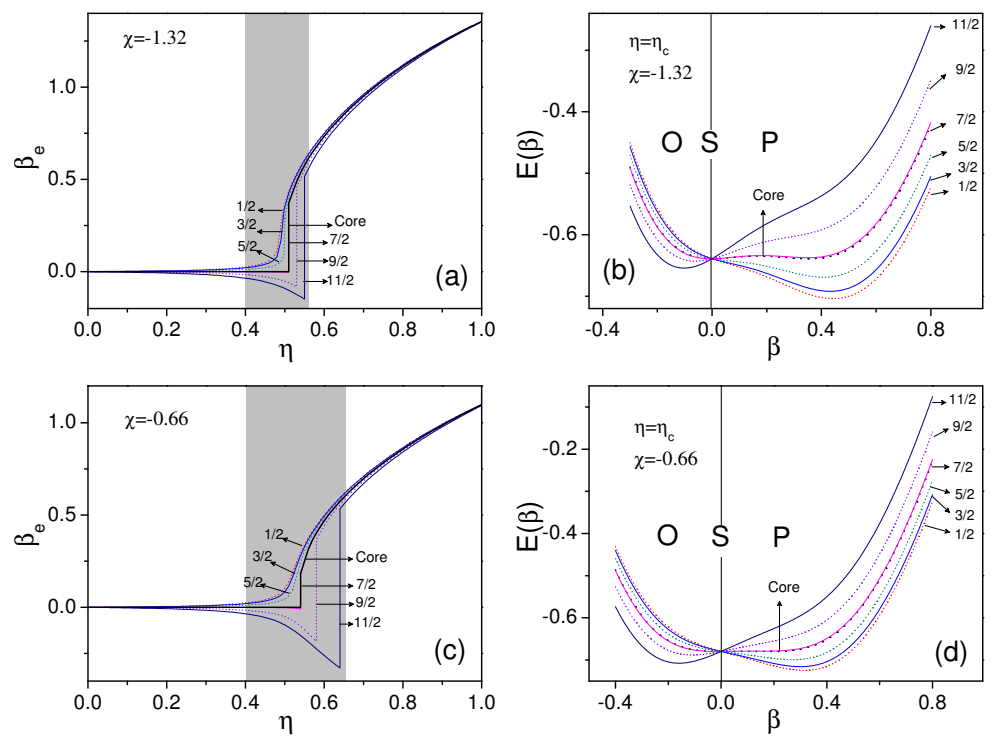

Figure 1. Evolution of the classical order parameter as a function of $\eta$ and the potential energy surface at the corresponding critical point in the cases with $\chi=-1.32$ and $\chi=-0.66$, respectively. $\mathrm{P}, \mathrm{O}$ and $\mathrm{S}$ represent prolate, oblate and spherical deformation.

(1) is taken into account. Since a single- $j$ shell is considered for the odd fermion, the pure fermion part contributes only an additional constant to the ground state energy [4].

To this end, we take the single fermion with $j=11 / 2$ as an example. Specifically, we adopt the intrinsic state for the coupled system $[4,5]$

$$
\beta, \gamma, N ; j, K\rangle_{B F}=\frac{1}{\sqrt{N !\left(1+\beta^{2}\right)^{N}}}\left[s^{\dagger}+\beta \cos \gamma d_{0}^{\dagger}+\frac{1}{\sqrt{2}} \beta \sin \gamma\left(d_{2}^{\dagger}+d_{-2}^{\dagger}\right)\right]^{N}|0\rangle_{B} \otimes a_{j K}^{\dagger}|0\rangle_{F} .
$$

to diagonalize the boson-fermion Hamiltonian (1) in the basis with $K=1 / 2,3 / 2, \ldots, j$ in order to get the energy eigenvalues $E(\beta, \gamma)$. Here, we consider, for simplicity, the case $\gamma=0^{\circ}$ for which the quantum $K$ would be a good quantum number $[4,5]$. If the effects of single fermion is analogous as an external field to the boson core, here $\gamma=0^{\circ}$ means the field with an axial symmetry [5]. Minimization of $E(\beta)_{K}$ with respect to $\beta$ for given $\chi$ and $\eta$ gives the optimal values $\beta_{e}$ with $\beta_{\mathrm{e}}=0, \beta_{\mathrm{e}}>0$, and $\beta_{\mathrm{e}}<0$ denoting the spherical, prolate, and oblate, respectively. Then, $\beta_{e}$ is considered as the classical order parameter to indicate order and type of the SPT. For a 1st order SPT, $\beta_{e}$ as a function of $\eta$ is not continuous. For a 2 nd order SPT, $\beta_{e}$ is continuous but $\frac{\partial \beta_{e}}{\partial \eta}$ is not. To perform a concrete analysis, the results calculated for $\chi=-1.32$ and $\chi=-0.66$ are shown in Fig. 1. As seen in Fig. 1(a), the results indicate that the effect of single fermion may weaken the phase transitional features for states with $K=1 / 2,3 / 2,5 / 2$ but enhance them for states with $K=9 / 2,11 / 2$, when comparing the cases for the even-even core. In contrast, the state for $K=7 / 2$ presents almost the same transitional feature as the one for the even-even core. It should be noted that these features were firstly revealed in $[5,6]$. More interestingly, one can find that a critical region (grey area in Fig. 1(a)), where $\beta_{e}$ for different $K$ suddenly changes with $\eta$, has been developed around the critical point $\eta_{c}$. It means that the critical behavior in the odd-even system can persist within a wider range of parameters in contrast 
to the adjacent even-even system. As seen from the Fig. 1(b), the potentials at $\eta_{c}$ for different $K$ have different configurations thus indicating different types of deformation or shape. Specifically, the cases for $K=1 / 2,3 / 2,5 / 2$ favor prolate deformation corresponding to $\beta_{e}>0$, while the cases for $K=9 / 2,11 / 2$ favor weakly oblate deformation corresponding to $\beta_{e}<0$. In contrast, the case for $K=7 / 2$ has a configuration as same as the one for even-even core, with two degenerate minima at $\beta=0$ and $\beta>0$, respectively. As further seen from Fig. 1(a), all the cases favor either the spherical $\left(\beta_{e}=0\right)$ or the prolate $\left(\beta_{e}>0\right)$ when $\eta$ runs outside of the critical region. It is thus concluded that the Bose-Fermi system may allow coexistence of different shapes only in the critical region, which in turn suggests that shape coexistence could be considered as a signature of the SPT in odd-even nuclei. Here, shape coexistence means that different types of deformation induced by both single-particle excitation and collective excitation may coexist in the low-energy structure at the same point $(\eta, \chi)$. In fact, it has been revealed that phenomenon of shape/phase coexistence indeed occurs in the lowlying spectrum of ${ }^{151} \mathrm{Sm}[8]$, which can be taken as the critical point nucleus in the spherical to prolate SPT [7]. Furthermore, one can find in Fig. 1(c) and (d) that the transitional features in the case with $\chi=-0.66$ are similar to those corresponding to $\chi=-1,32$ shown in Fig. 1(a) and (d), except that the critical region in the former is enlarged and shape coexistence becomes more pronounced due to the involvement of the $\mathrm{O}(6)$ components. Anyway, it suggests that there could be many more chances to identify phenomenon of shape coexistence in the Bose-Fermi system undergoing SPT.

\section{Summary}

In summary, a classical analysis of the shape phase transitions and the associated shape coexistence in odd-even nuclei have been performed within the framework of the IBFM. Specifically, we have analyzed the evolution of the deformations and the critical potential configurations in the case with the even-even core coupled to an single-fermion moving in the $j=11 / 2$ shell. The results indicate that the effects of odd particle may induce more pronounced shape coexistence in odd-even nuclei than in even-even nuclei around the critical point. Since only the quadrupole-quadrupole Bose-Fermi interaction term is considered in the IBFM Hamiltonian in the present discussion, other Bose-Fermi terms such as the exchange term and monopole term, which may potentially affect the shape phase transitional behaviors [6], should be included in the Hamiltonian to further prove the above conclusion.

\section{References}

[1] J. Jolie, S. Heinze, P. Van Isacker, and R. F. Casten, Phys. Rev. C 70, 011305(R) (2004).

[2] F. Iachello, Phys. Rev. Lett. 95, 052503 (2005).

[3] C. E. Alonso, J. M. Arias, L. Fortunato, and A. Vitturi, Phys. Rev. Lett. 98, 052501 (2007).

[4] M. Böyükata, C. E. Alonso, J. M. Arias, L. Fortunato, and A. Vitturi, Phys. Rev. C 82, 014317 (2010).

[5] F. Iachello, A. Leviatan and D. Petrellis, Phys. Lett. B 705, 379 (2011).

[6] D. Petrellis, A. Leviatan and F. Iachello, Ann. Phys. 326, 926 (2011).

[7] Y. Zhang, L. N. Bao, X. Guan, F. Pan, and J. P. Draayer, Phys. Rev. C 88, 064305 (2013).

[8] Y. Zhang, F. Pan, Y. X. Liu, Y. A. Luo, and J. P. Draayer, Phys. Rev. C 88, 014304 (2013).

[9] D. Bucurescu and N. V. Zamfir, Phys. Rev. C 95, 014329 (2017).

[10] K. Nomura, T. Nikšić, and D. Vretenar, Phys. Rev. C 96, 014304 (2017).

[11] F. Iachello and P. Van Isacker, The Interacting Boson-Fermion Model (Cambridge University Press, Cambridge, 1991) 